\title{
Printed interconnects for photovoltaic modules
}

\author{
J.D. Fields ${ }^{1}$, G. Pach ${ }^{1,2}$, K. Horowitz ${ }^{1}$, T.R. Stockert1,3, M. Woodhouse ${ }^{1}$, M.F.A.M. van Hest ${ }^{1}$ \\ ${ }^{1}$ National Renewable Energy Laboratory - 15013 Denver West Parkway, Golden, CO 80401 USA \\ 2 University of Colorado - Department of Electrical, Computer, and Energy Engineering, Boulder, \\ Colorado, 80309 USA \\ ${ }^{3}$ University of Wyoming - Department of Mechanical Engineering, Laramie, WY, 82071 USA
}

\begin{abstract}
Film-based photovoltaic modules employ monolithic interconnects to minimize resistance loss and enhance module voltage via series connection. Conventional interconnect construction occurs sequentially, with a scribing step following deposition of the bottom electrode, a second scribe after deposition of absorber and intermediate layers, and a third following deposition of the top electrode. This method produces interconnect widths of about $300 \mu \mathrm{m}$, and the area comprised by interconnects within a module (generally about $3 \%$ ) does not contribute to power generation. The present work reports on an increasingly popular strategy capable of reducing the interconnect width to less than $100 \mu \mathrm{m}$ : printing interconnects. Cost modeling projects a savings of about $\$ 0.02 /$ watt for CdTe module production through the use of printed interconnects, with savings coming from both reduced capital expense and increased module power output. Printed interconnect demonstrations with copper-indium-gallium-diselenide and cadmiumtelluride solar cells show successful voltage addition and miniaturization down to $250 \mu \mathrm{m}$. Material selection guidelines and considerations for commercialization are discussed.
\end{abstract}

Keywords: Thin-film photovoltaics; module construction; monolithic interconnects; printing

\section{Introduction}

Film-based photovoltaic (PV) technologies, such as cadmium telluride (CdTe), copperindium-and copper-indium-gallium- diselenide (CIS and CIGS), amorphous silicon, and the various types of organic polymer:fullerene systems (e.g. P3HT:PCBM), employ monolithic interconnects (ICs) to add the voltage between cells in modules. Conventional IC construction occurs sequentially, with a scribing step following deposition of the bottom electrode, a second scribe after deposition of absorber and intermediate layers, and a third following deposition of the top electrode - the so-called P1, P2, and P3 scribes, respectively (Fig. 1). The area comprised by ICs does not contribute to power generation, and therefore, a trade-off applies between the module geometric fill-factor and the IC width. A module with narrower ICs contains more active area, and therefore generates more power. The present work reports on an increasingly popular strategy to reduce the IC width and at the same time streamline module assembly: printing ICs. Consider the cumulative impact of $300-\mu \mathrm{m}$-wide ICs on a module with cell width of $1 \mathrm{~cm}$ (characteristic for CIGS and CdTe PV): this amounts to $3 \%$ dead area; for a $200 \mathrm{~W}$ module more than $5 \mathrm{~W}$ of possible energy generation is forgone. 


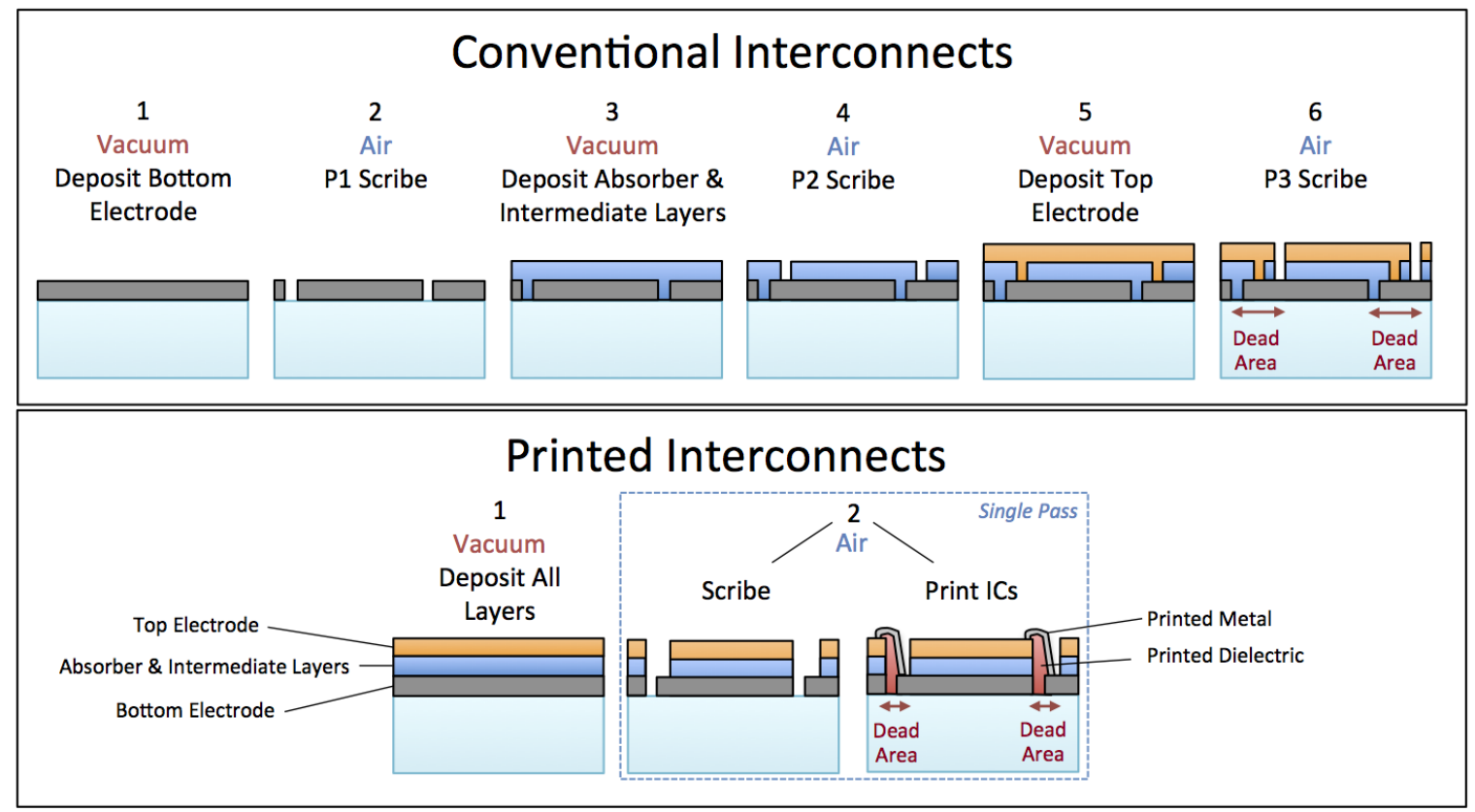

Fig. 1. Process sequence for monolithic cell integration using (top) conventional vs. (bottom) printed interconnects (2-scribe geometry depicted).

Previously, IC width reduction has been achieved by reducing the width of individual scribe lines. Here, transitioning from ns-pulsed lasers to ps-pulsed lasers for ablation provides advantages, enabling both narrower and more uniform scribing due to reduced thermal effects [1-5]. This also reduces unintended deposition of ablated materials on scribe sidewalls, which unmitigated create shunting pathways [4,5]. Other authors report IC width down to $250 \mu \mathrm{m}$ using a combination of pulsed-laser and optimized mechanical scribing [6]. Additional IC width reduction was achieved by replacing P2 and P3 mechanical scribing with ps-laser scribing. In this way other researchers achieve IC widths down to $200 \mu \mathrm{m}[3,7]$. These approaches will provide short-term improvements for thin-film PV manufacturers (current IC width $\sim 300 \mu \mathrm{m}$ ). However, to go below $200 \mu \mathrm{m}$ will likely require alternative IC architectures, and the printed IC approach provides the means.

Although Thalheimer patented the original printed IC process almost 30 years ago, the method only recently finds traction in mainstream PV manufacturing [8]. Renewed interest in printing the ICs is evidenced by numerous patents filed in the last several years in this area [9-12] and multiple developmental efforts launched by collaborative technology consortiums $[13,14]$. The increased attention follows key advances in direct-write, printedelectronics technologies in the last several decades. Commercially available lowtemperature (less than $200{ }^{\circ} \mathrm{C}$ ) and UV-curable inks enable deposition of conductive and dielectric IC features with minimal thermal-induced degradation of solar cell materials, improved printing resolution (feature size down to $20 \mu \mathrm{m}$ ) allows IC patterning with minimal shading, and industry relevant deposition rates now provide the means for implementation of direct-write processes in high-volume manufacturing $[15,16]$. Couple these improvements with a modern laser-scribing practice, and printing ICs with nontraditional architectures becomes a viable option.

In addition to providing higher geometric fill-factor, printing ICs after deposition of all solar cell layers provides many other advantages. For one, performing one scribing/interconnection step, as opposed to performing three separate P1-, P2-, P3scribing steps at different stages of the solar module production sequence, eliminates 2 
process steps and streamlines production. Integrated scriber/printer instruments, such as those reported in Refs. [10] and [14] can accomplish this. The method also eliminates venting and pumping time delays between vacuum-/atmospheric-pressure-based process steps associated with ex-situ transfers in the conventional process (see Fig. 1), wherein exposure to air between deposition steps can degrade cell performance, such as by oxidation for example. Thus, enabling complete cell deposition under vacuum before transferring to atmosphere for scribing may also enable additional power gains. Lastly, in the case of CIGS, since the Mo is freshly exposed for interconnection in a printed IC process, parasitic contact resistance between the TCO and molybdenum (Mo) bottom electrode associated with a deleterious molybdenum-diselenide intermediate layer is avoided [17]. Hence, printing the ICs provides numerous paths to power gains.

This work focuses on material selection considerations for printing ICs, and the expected impact of implementing a printed IC process on CdTe PV module manufacturing costs. Printed IC demonstrations with both CIGS and CdTe modules show that this strategy can be applied to both substrate and superstrate configuration solar cells, analogously. Cost modeling weighs expected benefits of printed ICs against estimated capital investment required to implement a printed IC process in CdTe PV manufacturing. Calculations project a savings of about $\$ 0.02 /$ watt. Key in favorable manufacturing economy is higher module power with comparable capital expense since the additional cost of printing equipment is offset by savings achieved by reducing the number of steps and eliminating ex-situ transfers.

\section{Printed Interconnect Architectures}

Numerous IC architectures, including the conventional 3-scribe form, are possible through the use of a printed dielectric and printed metal to make series connections between cells. A printed 3-scribe IC (Fig. 2(top)) is made by performing a P1-scribe through the full stack, P2- and P3-scribes down to the bottom electrode, and then depositing dielectric and metal into the scribes. Here, the dielectric printed into the P1 scribe isolates the bottom electrode upon subsequent metallization to make the series connection between two cells. However, examining the printed 3-scribe IC form, with printed dielectric and metal features separated by absorber/TCO stack margins, one recognizes the P1/2 and P2/3 margins need not be maintained to make the electrical series connection and also maintain electrical isolation of top and bottom electrodes. Fig. 2(middle) shows a 2-scribe IC architecture (demonstrated in Section 4), made by performing a P1-scribe through the full stack, a P2-scribe to expose the bottom electrode, and then printing carefully so as to maintain the margin between the printed metal and the P2-scribe side-wall. Ultimately,

with sufficient control over the dielectric and metal printing resolution, and with optimal alignment of the deposition to the P1-scribe, a single-scribe IC should be possible. This concept is shown in Fig. 2(bottom). While the single-scribe IC remains an elusive goal experimentally, it should be possible in principal. 

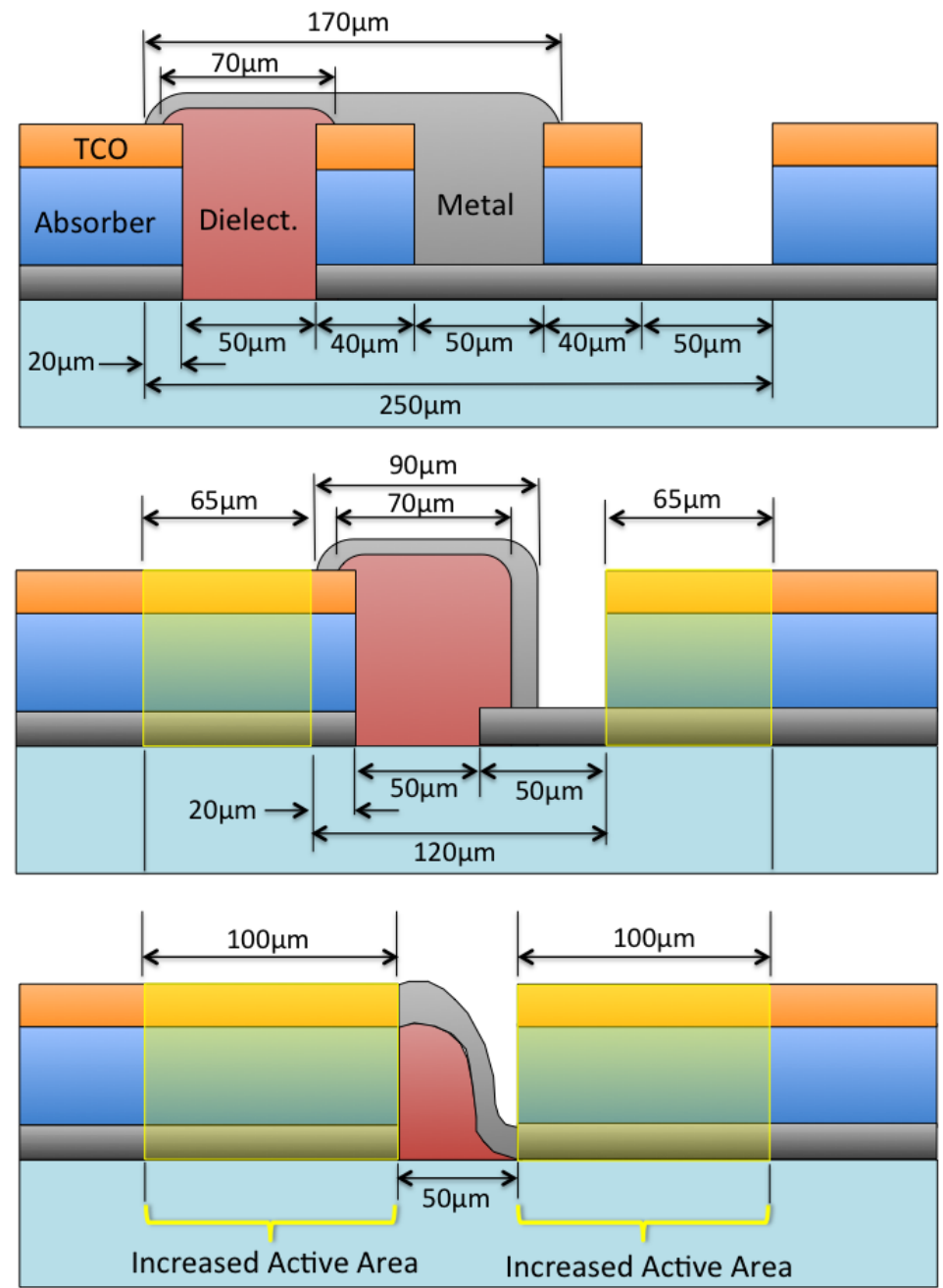

Fig. 2. 3-scribe, 2-scribe, and 1-scribe printed IC architecture concepts (top, middle, and bottom, respectively).

With increasingly narrow scribe-widths possible using new ultrafast lasers (scribe widths approaching $10 \mu \mathrm{m}[3]$ ), and improved industrial printing capabilities, the opportunity to integrate scribing and printing tools to reduce the 3 -scribe IC structure width to $250 \mu \mathrm{m}$ becomes attainable. In the case shown in Fig. 2(top) the IC-width is limited by scribe widths of $\sim 50 \mu \mathrm{m}, \mathrm{P} 1 / 2$ and P2/3 margin widths of $40 \mu \mathrm{m}$, and printed feature width down to $70 \mu \mathrm{m}$. Here, $40-\mu \mathrm{m}$-wide margins suffice, as opposed to wider margins on the order of 100- $\mu \mathrm{m}$-wide in conventional IC's, since the scriber and printer are integrated in a printed-IC process, which reduces the risk of shunting. IC width approaching $100 \mu \mathrm{m}$ should be attainable by printing ICs with simplified architectures. Fig. 2(middle) shows the feature sizes needed to construct a 2-scribe IC with total width of just $120 \mu \mathrm{m}$, adding more than $100 \mu \mathrm{m}$ of active cell width. Despite limitations on the ablation process quality in the present work, discussed in Section 4, IC-widths down to $250 \mu \mathrm{m}$ could still be obtained using a variation on the 2-scribe IC architecture. It is also clear that miniaturization to less than $100 \mu \mathrm{m}$ should be possible using existing technology.

\section{Material selection}


This section explains the selection criteria for identifying suitable dielectric and metal inks for printing ICs to make CIGS and CdTe PV modules, which was accomplished using an Optomec aerosol-jet system for convenience. The printing speed was $10 \mathrm{~mm} / \mathrm{s}$ in all experiments, which is slow by industry standards. However, findings in this work are readily transferable to other printing techniques with higher deposition speeds, such as inkjet printing, but ink formulation and viscosity must be optimized to achieve consistent printing in this case. The Optomec aerosol-jet system used in the present work accepts a wide range of ink viscosities ( 1 to $1000 \mathrm{cp}$ ), and enables contact-free deposition of lines down to $10 \mu \mathrm{m}$ via pneumatic atomization and nitrogen gas column-focused, ultra-fine spray. Substrate configuration CIGS cells used in this work (mixed cell efficiency ranging from $4 \%$ to $9 \%$ ) consisted of a 1- $\mu$ m-thick sputtered molybdenum (Mo) bottom electrode, 2- $\mu \mathrm{m}$ thick evaporated CIGS absorber, 80-nm-thick of cadmium-sulfide (CdS), 50-nm-thick sputtered intrinsic zinc-oxide ( $\mathrm{ZnO}$ ), and about $500-\mathrm{nm}$ of aluminum-doped zinc-oxide (AZO) as the transparent conducting oxide (TCO). Superstrate configuration CdTe solar cells used in this work (efficiency about $10 \%$ ) are described in detail in Ref. [18]. Generally, the CdTe cells had a MOCVD-deposited bilayer of fluorine-doped tin-oxide (FTO) and intrinsic tin-oxide as the TCO (sheet resistance $\sim 15 \Omega / \square)$, reactively sputtered CdS $(100$ nm-thick), close-spaced sublimed CdTe as the absorber layer (4- $\mu \mathrm{m}$-thick), co-evaporated copper-doped zinc-telluride (Cu:ZnTe) (150-nm-thick), and evaporated gold (Au) top contacts.

\subsection{Dielectric Inks for Printed Interconnects}

Dielectric material printed into the P1 scribe provides electrical isolation between cells (see Figure 2). Since inadequate dielectric coverage results in shunting when metal is printed to make the series connection, reliable and controlled deposition of a robust dielectric is paramount for making printed ICs. Criteria considered for determining the suitability of a dielectric-ink for use in printed ICs include:

(i) Resistivity - the dielectric must have sufficiently high resistivity to prevent shunting between cells,

(ii) Compatibility - neither the ink curing temperature nor the solvent may degrade the solar cell materials or device functionality,

(iii) Interface characteristics - the ink must exhibit good wetting characteristics with the solar cell materials (i.e. favorable interface energy), and adhesion strength between the dielectric and the material needing isolation,

(iv) Ease of printing - resolution of printed features, nozzle clogging, and ink stability

(v) Durability and mechanical strength - the dielectric must possess sufficient tensile and compressive strength to accommodate thermal expansion and contraction both during module construction and in the field during the lifetime of the PV module.

Of the various dielectric inks investigated in the present work, poly(methyl-methacrylate) (PMMA) produced the best results. Aerosol-jet deposited PMMA (5 wt. \% in acetophenone), printed at a substrate temperature $\left(\mathrm{T}_{\mathrm{s}}\right)$ of $100{ }^{\circ} \mathrm{C}$ and cured at $200{ }^{\circ} \mathrm{C}$, exhibited resistivity of about $10^{4} \Omega$-cm in metal-insulator-metal test structures. This level of electrical isolation was sufficient for the present work. This PMMA ink also demonstrated good wetting 
properties. A contact angle of about $10^{\circ}$ to $15^{\circ}$ was observed for acetophenone (the PMMA ink solvent) for both the top and bottom electrode materials in the CIGS solar cells (i.e. AZO or Mo), and addition of PMMA did not impact the wetting properties (see Supplementary Material, Fig. S1). Wetting of the PMMA ink on CdTe cell electrode materials (Au and FTO) was also favorable. The ink was also easy to use. Narrow PMMA lines or pads - features needed for IC construction - were readily achievable by aerosol jetting (see Supplementary Material Fig. S2), and nozzle clogging was not an issue, even with intermittent printing over multiple days, and without cleaning the hardware between depositions.

In the case of substrate configuration CIGS, the condition of solvent compatibility concerns the top electrode (typically a TCO), the CdS buffer layer, and the absorber. If the solvent dissolves or compromises any of these layers, then cell performance may suffer. This was not observed with the PMMA/acetophenone dielectric ink, evidenced by comparing the performance of cells after vs. before deposition of PMMA into P1 scribes (Fig. 3). Slight reductions in open-circuit voltage $\left(\mathrm{V}_{\mathrm{oc}}\right)$ and short-circuit current density $\left(\mathrm{J}_{\mathrm{sc}}\right)$, after PMMA deposition are accounted by minor reflection and shading effects from having narrow strips of dielectric printed over active area on both sides of the cell. Additionally, flat I-V characteristic at $1 \mathrm{~V}$ reverse-bias confirms a high shunt resistance is retained after interaction with the PMMA and solvent. Analogously prepared CdTe samples showed no degradation after depositing PMMA. Therefore, while higher efficiency devices may be more sensitive and demand more sensitive inks, compatibility of the PMMA ink was confirmed for CIGS and CdTe cells used in the present work.

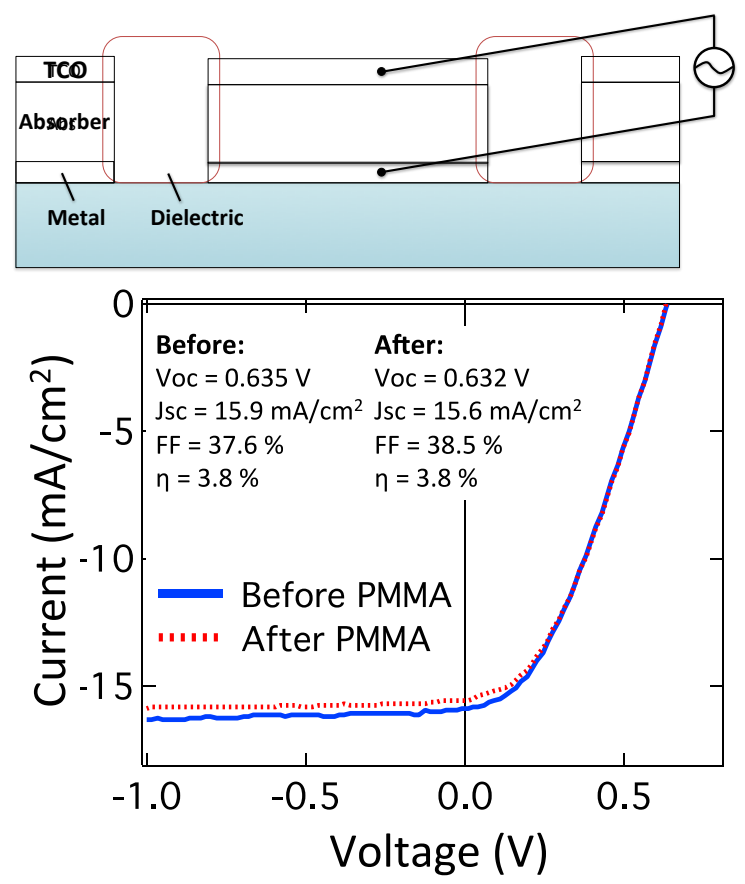

Fig. 3. Sample geometry for evaluating printed-PMMA process compatibility (top), and corresponding $\mathrm{J}-\mathrm{V}$ characteristics of a CIGS solar cell measured pre- vs. post-deposition. Pre-metallization IV measurement utilized a small piece of indium attached to the probe tips to contact the cell gently and avoid damage.

A variety of dielectric materials are potentially useful for printed ICs. Similarly other methods besides aerosol-jet deposition may be used - for example ink-jet printing [10] and screen-printing [8]. Additional dielectric materials examined in this work include poly-4- 
vinyl-phenol (PVP), and select low temperature siloxane glass materials (i.e. low-viscosity spin-on-glass type formulations amenable to print-processing, with relatively low curing temperatures of $\sim 200{ }^{\circ} \mathrm{C}$ ). Indeed, superior chemical stability of inorganic, ceramic dielectric materials makes them attractive in this application. However, these inks were not specifically optimized for printing and so did not print well, depositing too thick and frequently clogging the nozzles. Moreover, the resulting $\mathrm{SiO}_{\mathrm{x}}$ layers tended to crack during cooling or accompanying temperature cycling during metal deposition due to and weak tensile strength. On the other hand, higher toughness of organic dielectric materials better accommodates thermal expansion and contraction. While, all cells used in this work were on rigid, glass, substrates, the observed tendency of $\mathrm{SiO}_{\mathrm{x}}$ to crack under minimal strain suggests organic dielectrics may be better suited for flexible PV applications, which demand even higher tensile strength to accommodate bending. Since the PMMA/acetophenone ink printed reliably and gave smooth, continuous, dielectric features with fair insulating quality, this material was used to demonstrate printed ICs.

\subsection{Metal-Inks for Printed Interconnects}

Selection of metal-inks for use in printed IC construction follows the same compatibility and printing reliability criteria specified in the Dielectric-Inks discussion, with the additional requirement that the resulting printed line have low electrical resistivity within the allowable process temperature range - again, ideally below about $200{ }^{\circ} \mathrm{C}$. Both metalorganic- and nanoparticle-based silver (Ag) and nickel (Ni) inks meet this lowtemperature/low-resistivity requirement. However, some of the inks examined in the present work failed acceptance testing for solvent/chemical compatibility and/or printing reliability issues. Printing of trident-contact patterns onto CIGS and CdTe test cells (Fig. 4) served as the primary test for material compatibility and ease of printing. If a trident pattern could not be made repeatedly (i.e. printed onto a series of samples with start and stops to allow for sample changes and heating), then the ink failed acceptance testing. It follows that the ensuing discussion only considers inks found to print reasonably well or without issue.

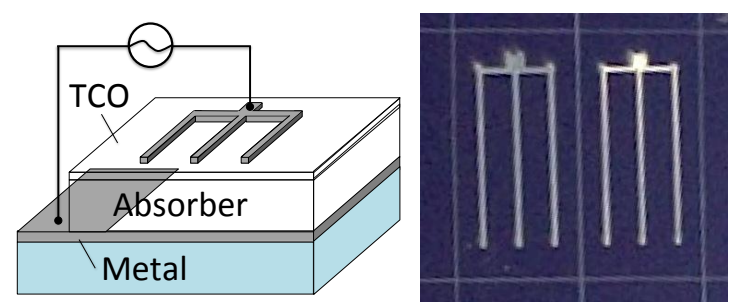

Fig. 4. (left) Printed metal-ink test structure, and (right) photograph showing two CIGS test cells ( $5 \mathrm{~mm} \times 9 \mathrm{~mm}$ ) with printed Ag trident contacts.

Comparing the current density vs. voltage (J-V) characteristics of CIGS cells before vs. after metal contact printing provides an indication of ink compatibility. As shown in Fig. 5, a cell contacted using only a small piece of indium metal underneath the contact-probe shows modest performance for a CIGS cell, with $\mathrm{V}_{\mathrm{oc}}$ of $0.616 \mathrm{~V}$, $\mathrm{J}_{\mathrm{sc}}$ of $26.2 \mathrm{~mA} / \mathrm{cm}^{2}$, FF of $42.4 \%$, and efficiency of $6.85 \%$ (top-left). The Ag and Ni metalorganic decomposition inks (topright) induced what appears to be shunting, whereas cells contacted with Ag nanoparticle inks maintained their performance or demonstrated an improvement through the effect of higher FF (bottom-left and bottom-right). 

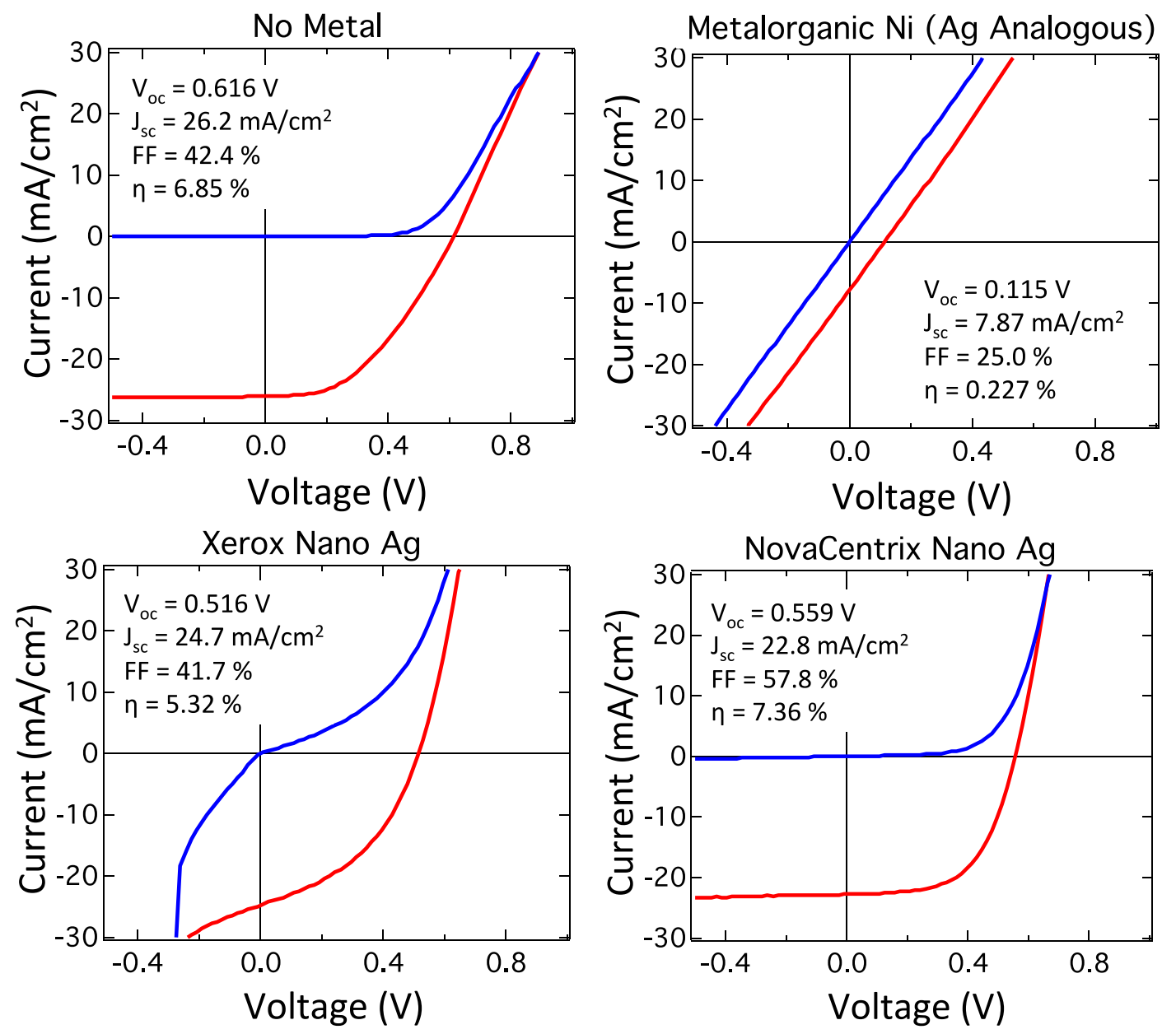

Fig. 5. Light and dark J-V traces measured from CIGS solar cells contacted with (a) no metallization, (b) metalorganic Ni printed at $180{ }^{\circ} \mathrm{C}$, (c) Xerox Ag nanoparticle ink printed at $150{ }^{\circ} \mathrm{C}$, and (d) NovaCentrix Ag nanoparticle ink printed at $150^{\circ} \mathrm{C}$.

Cells contacted with the Xerox and NovaCentrix Ag nanoparticle inks (product IDs: XCMnsPA and HPS-030AE1, respectively) performed significantly better than those contacted with metalorganic Ag and Ni inks. The cell contacted with Xerox nano-Ag ink retained $\mathrm{J}_{\mathrm{sc}}$ and fill-factor values comparable to the control cell. However, the $\mathrm{V}_{\text {oc }}$ degraded by approximately $0.1 \mathrm{~V}$ upon metallization, and the cell showed an appreciable leakage current under reverse bias. This is not to say that all CIGS solar cells will shunt using Xerox Ag inks, but rather the CIGS materials used in this study specifically showed this tendency using this specific formulation. On the other hand, the cell contacted with NovaCentrix nano-Ag ink showed the best performance, with an increase in photoconversion efficiency from $6.9 \%$ in the control sample to $7.4 \%$ in the contacted sample. This gain reflects an increase in fillfactor, from $42 \%$ to $58 \%$, due to reduced series resistance in the top electrode/contact. However, the $\mathrm{V}_{\mathrm{oc}}$ also decreased slightly with this ink, from $0.61 \mathrm{~V}$ to $0.56 \mathrm{~V}$, and the $\mathrm{J}_{\mathrm{sc}}$ decreased from $26 \mathrm{~mA} / \mathrm{cm}^{2}$ to $23 \mathrm{~mA} / \mathrm{cm}^{2}$. The $\mathrm{J}_{\mathrm{sc}}$ reduction can be attributed to shading; the cause of $V_{\text {oc }}$ loss, however, remains in question. Regardless, the CIGS cells used in this experiment showed the best compatibility with the nanoparticle inks.

Both of the Ag nanoparticle inks printed well using the Optomec aerosol-jet deposition system - occasionally clogging occurred, but this was generally the result of prolonged 
stoppage (e.g. after an overnight idle) which allowed ink drying within the nozzle. With minimal start/stoppage, printing could run without issue for about 5 hours straight, and then a simple nozzle change would allow resumed deposition. These nano-metal inks also delivered impressively low resistivity values of $5 \times 10^{-6} \Omega$-cm for the Xerox ink, with $T_{s}=$ $130^{\circ} \mathrm{C}$ and $1 \times 10^{-5} \Omega$-cm for the NovaCentrix ink with $\mathrm{T}_{\mathrm{s}}=150^{\circ} \mathrm{C}$ (note: $\rho_{\mathrm{Ag}, \mathrm{bulk}}=1.6 \times 10^{-6} \Omega$ cm under STC).

Although though the Xerox ink provided lower resistivity and at a lower temperature compared to the NovaCentrix ink, the NovaCentrix ink was used for IC experiments because it did not induce the high reverse-bias current as observed with the Xerox ink, and it printed slightly more consistently. In addition, the NovaCentrix ink is water-based allowing for easier handling and cleaning, whereas the Xerox formulation contains an organic solvent.

In the present work identifying a compatible metal-ink for use with the CIGS solar cells presented a challenge. However, this does not apply generally. In a previous publication by some of the present authors, some other CIGS solar cells showed excellent compatibility with a metalorganic Ag ink formulation that was analogous to that used in the present work [19], indicating that variations in CIGS morphology can lead to different response to inks. On the other hand, the CdTe cells passed all metal-ink compatibility tests regardless of ink formulation, nature (nanoparticle vs. metalorganic), or curing temperature (note: all tests were done with $\mathrm{T}_{\mathrm{s}}$ less than $250{ }^{\circ} \mathrm{C}$ ), and therefore this system seems to be more amenable to solution processing. It remains to be seen, however, whether the better tolerance follows from the CdTe cells having superstrate configuration with metal encapsulation on top, compared to CIGS cells having substrate configuration with a TCO top layer, or whether the CdTe stack materials are simply more stable with exposure to solvents.

\section{Mini-Module Experiments}

\subsection{Mini-Module Construction}

Printed IC demonstrations utilized both laser- and mechanical-scribing. Full-stack, P1laser-scribing employed a Q-switched, Nd:YAG laser, tuned to $532 \mathrm{~nm}$, with 1.3 -watts RMS power, 8-ns pulses, 15-kHz repetition rate, and a beam diameter of $\sim 40 \mu \mathrm{m}$. Full-stack mechanical scribing for cell isolation was done with a diamond tip. P2 and P3 mechanical scribes were made using a tungsten-carbide tip, producing scribe widths of approximately $100 \mu \mathrm{m}$. In experiments with CIGS, a tungsten-carbide tip was also used to dress P1-scribe sidewalls after laser-scribing, as reported in Ref. [13], which made the total P1-scribe width about 250 to $300 \mu \mathrm{m}$. Cleaning the sidewall was required to remove metal deposits formed during P1-laser-scribing, which when unmanaged act as shunting pathways. Full-stack scribing with a ps- or fs-pulsed laser will avoid this issue, as under optimized conditions these faster lasers remove material by thermomechanical fracture rather than ablative evaporation, as reported by others [2-4,20]. To make 3-scribe ICs, PMMA was deposited at $100{ }^{\circ} \mathrm{C}$ into the $\mathrm{P} 1$-scribe, and then after dielectric printing, the samples were P2-scribed and loaded back into the aerosol-jet system for metallization. For 2-scribe ICs, the P2-scribe was made before PMMA printing, and connected to the P1 scribe (Fig. 6). Ag deposition at $150{ }^{\circ} \mathrm{C}$ over the dielectric in the P1 scribe and down to the lower electrode exposed by the P2 scribe makes the series connections. In 3-scribe ICs, P3-scribes were then made using tungsten-carbide tip. Else, for 2-scribe ICs a margin maintained between the Ag and P2 scribe side-wall maintains electrical isolation. 


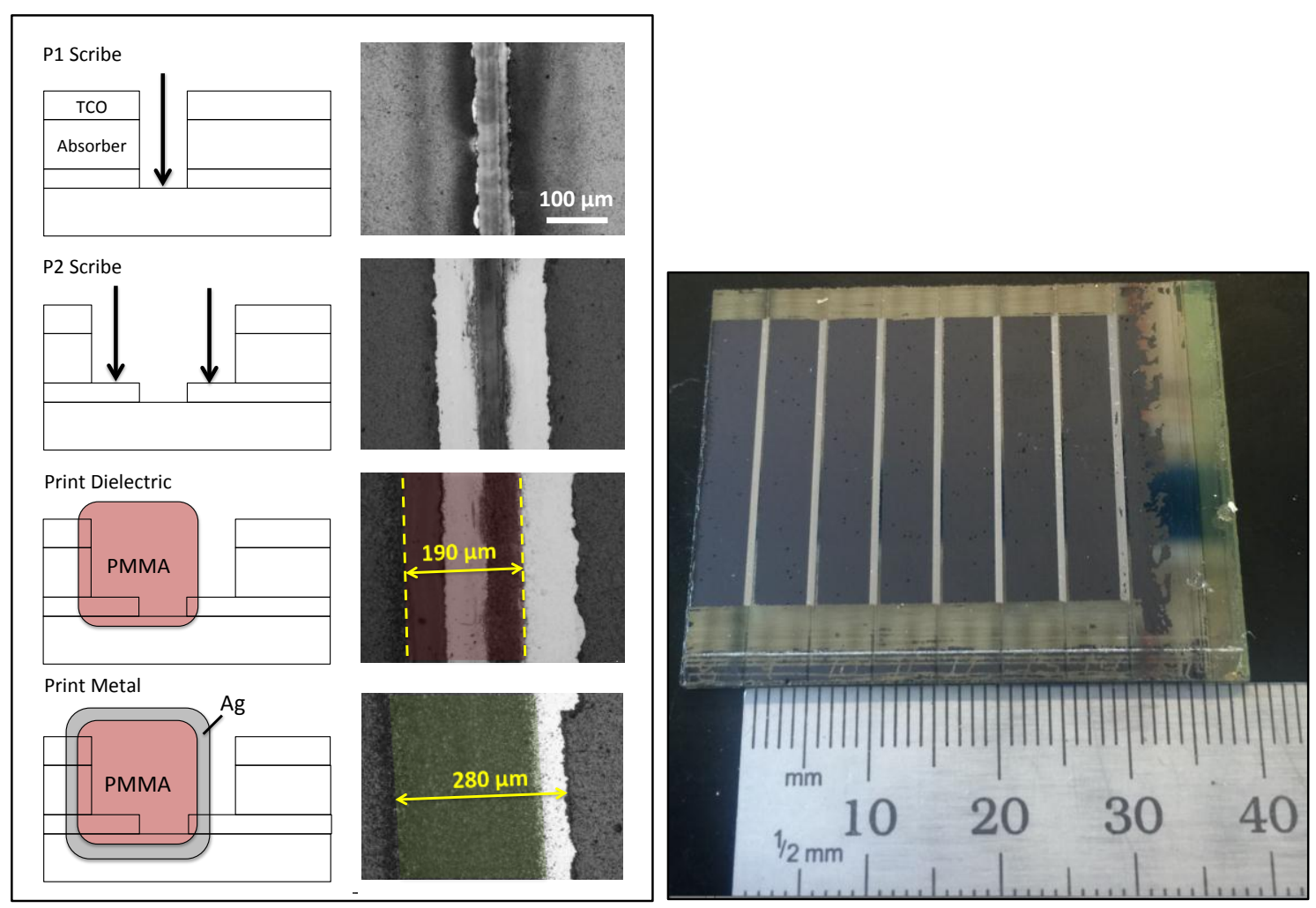

Fig. 6. P1/P2 printed IC construction sequence shown schematically, and with microscope images (left), and a photograph of the interconnected mini-module with $250 \mu \mathrm{m}$-wide ICs (right)

\subsection{Electrical Characterization and Discussion}

Series connected CIGS mini-modules, made with printed ICs using PMMA and NovaCentrix Ag inks, show successful voltage addition (Fig. 7, left). Light and dark J-V traces from a single cell up to 5 interconnected cells are shown. A string of 5 CIGS cells produced a $V_{\text {oc }}$ of $2.86 \mathrm{~V}$, with each cell contributing over $0.56 \mathrm{~V}$. Similarly, 4 interconnected CdTe cells gave $2.53 \mathrm{~V}$ (Fig. 7, right). Voltage addition was less than ideal in the CdTe module, evidenced by incremental voltage additions lower than the $0.8 \mathrm{~V}$ expected for each cell. The first cell in the CdTe string delivered the expected $\mathrm{V}_{\text {oc }}$ of $0.802 \mathrm{~V}$, but the next cell only added $0.478 \mathrm{~V}$, and adding cells 3 and 4 only increased the voltage by similar increments. It appears the ICs in this module impart parasitic resistance in the string and consume about $0.3 \mathrm{~V}$ per cell. The fill-factor also suffered appreciably with increasing number of cells in the CdTe module, beginning $61 \%$ for 1 cell and decreasing to $43 \%$ for 4 cells. On the other hand, the CIGS module exhibits a fill-factor of about $40 \%$, irrespective of the number of interconnected cells. 

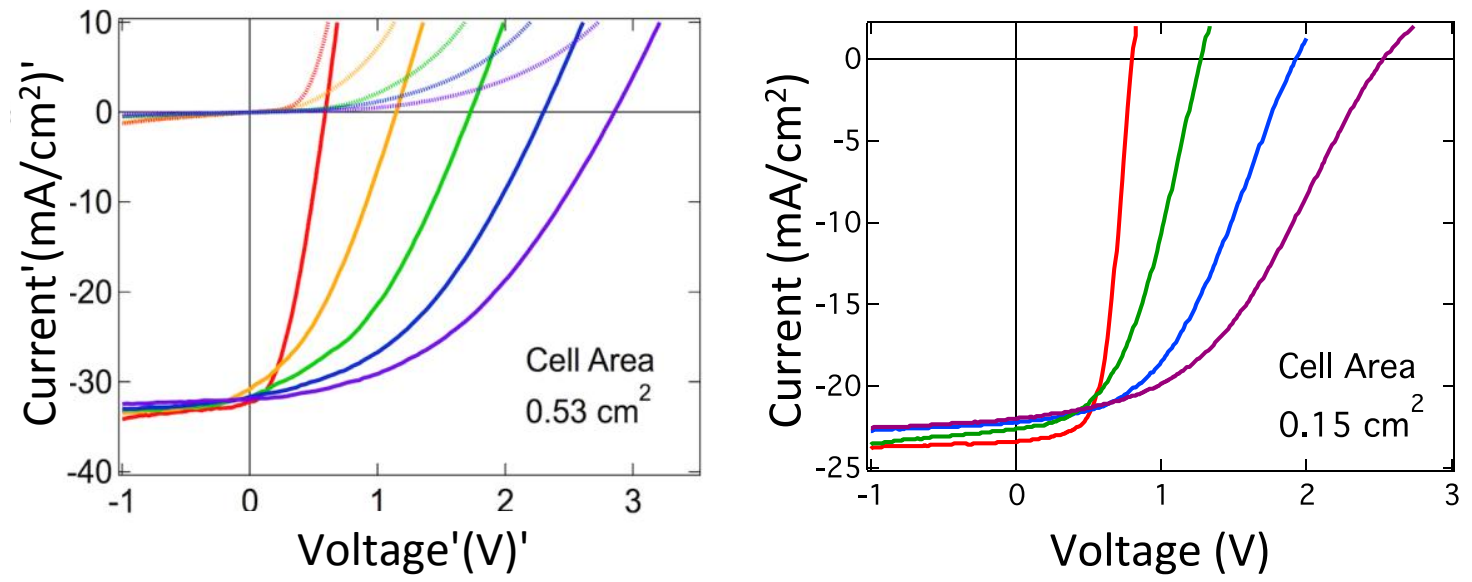

Fig. 7. J-V characteristics of interconnected CIGS and CdTe cells (left \& right, respectively) with metalbridged 3-scribe ICs. (Note: Jsc expressed in terms of active area. See Fig. S2 in Supplementary Information for details).

Additional evidence of module performance limited by series resistance $\left(\mathrm{R}_{\mathrm{ser}}\right)$ considers the systematically decreasing slope of $\mathrm{J}-\mathrm{V}$ curves in forward bias with increasing number of cells. Approximating the magnitude of $\mathrm{R}_{\mathrm{ser}}$ by the slope at $\mathrm{V}_{\text {oc }}$ suggests a series resistance of $100 \Omega$ in the CIGS module with 5-cells, and over $500 \Omega$ in the string of 4 CdTe cells. Sheet resistance in the TCO materials is a likely culprit here, which was lower for AZO $(\sim 10 \Omega / \square)$ in the CIGS stack compared to FTO $(15 \Omega / \square)$ in the CdTe stack. The TCO layers can be made thicker to reduce the sheet resistance, or in the case with CIGS modules, metal fingers could be added perpendicular to the ICs. However, since both of these will result in some added optical loss (believe on the order of $\sim 1 \mathrm{~mA} / \mathrm{cm}^{2}$ reduction in $\mathrm{J}_{\mathrm{sc}}$ ) the TCO thickness must be optimized to maximize FF ${ }^{*}$ Jsc (see $[17,21]$ ). The balance between minimizing resistive-loss and optical-loss associated with the front-contact/top-electrode/emitter is universal, applies for all PV technologies (excepting all-back-contact devices), and is not unique to modules with printed ICs. The decision of whether to add a metal grid or increase the TCO thickness to minimize series resistance depends on the application. In some cases, such as for flexible PV devices, employing a relatively thick, low sheet-resistance, TCO may not be an option because this would lead to stiffening, and hence, brittle failure of the topelectrode accompanying strain. Here, a metal grid with superior toughness and tolerance to plastic deformation may be better suited. On the other hand, for rigid-PV devices, such as glass-encapsulated modules, the optimal stack may possess a thicker, high \% transmission, TCO, allowing low series resistance and with minimal optical loss.

A module with 2-scribe ICs (see Fig. 2 - center) demonstrates an initial step towards miniaturization. This design can produce an IC width down to $\sim 150 \mu \mathrm{m}$ wide by printing the dielectric and metal features with diameter of about $50 \mu \mathrm{m}$ and $100 \mu \mathrm{m}$, respectively, and allowing a $50-\mu \mathrm{m}$-wide margin between the metal and scribe side-wall - all possible using existing technology. A proof of concept module build using $\sim 250 \mu \mathrm{m}$ IC width shows perfect voltage addition with increasing number of cells up to a $\mathrm{V}_{\text {oc }}$ of $3.96 \mathrm{~V}$ for 6-cells (Fig. 8), and $\mathrm{FF} \sim 40 \%$. 


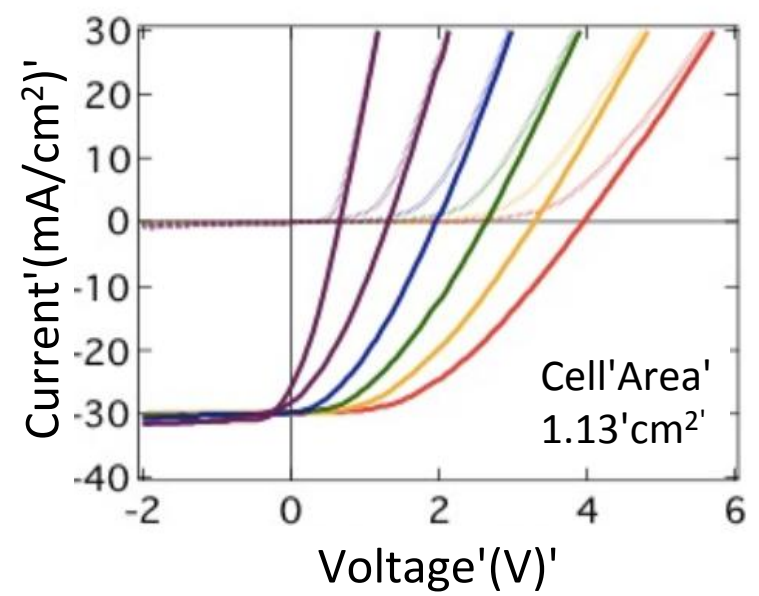

Fig. 8. J-V characteristics of CIGS cells series connected with 2-scribe ICs.

\subsection{Limitations}

While these module demonstrations suffice for proof-of-concept, there is room for improvement. For one, the electrical performance can be enhanced through the use of a dielectric with higher resistivity. As previously stated, the PMMA $\left(\rho \cong 10^{4} \Omega-\mathrm{cm}\right)$ enabled successful demonstrations of printed ICs. However, some of the interconnected strings suffered from leakage current under reverse bias, which reduces the open-circuit voltage by the dependence specified in Eq. 1.

$$
V_{o c}=\frac{n k T}{q} \ln \frac{I_{L}}{I_{0}} \div \quad \text { Eq. (1) }
$$

In Eq. $1, n$ is the diode ideality factor, $k T / q$ is the thermal voltage, $I_{L}$ is the light-generated current, and $I_{0}$ is the saturation reverse current. Shunting through the dielectric contributes to the saturation reverse current, $I_{0}$. Printed PMMA demonstrated a resistivity of about $10^{4}$ $\Omega$-cm; the expected leakage current for $2 \mu \mathrm{m}$ thick dielectric at $1 \mathrm{~V}$ is about $5 \mathrm{~mA} / \mathrm{cm}^{2}$. Thus, replacing PMMA with a more resistive material would be helpful for optimizing performance as this will reduce $I_{0}$ and raise the $V_{\text {oc }}$ of interconnected strings to their full potential. In addition, while the Ag nanoparticle inks showed by far the best performance in compatibility testing (Section 3), the $\mathrm{V}_{\text {oc }}$ of CIGS cells still degraded partially from the full $620 \mathrm{mV}$ to $<600 \mathrm{mV}$, even with the best ink evaluated in the present study. Thus, additional improvement may be realized by optimizing the metal ink formulation.

\section{Cost Considerations and Commercialization}

Printing ICs in thin-film PV manufacturing offers a cost reduction. Detailed cost analysis considers both 2-scribe and 1-scribe printed IC processes (see Fig. 2), with miniaturization of the IC width down to $100 \mu \mathrm{m}$ and associated costs itemized for each step in CdTe-based PV module production. The model considers an industrial ink-jet printer integrated with a high-throughput, ns-pulsed, P1/P2 laser scriber. Scribing and printing are assumed to occur at the same rate, in a single pass with the laser leading a print head to deposit PMMA 
into the scribe, and an additional print-head used to deposit Ag over the insulator and connect with the lower electrode (see Fig. 2 - bottom). Cost analysis with printed-ICs assumes substrate temperature of $150{ }^{\circ} \mathrm{C}$ for ink curing, achieved with an integrated, $50 \mathrm{~kW}$ ceramic heater. The power rating quoted to obtain this target temperature is based on interviewers with furnace suppliers, and the energy cost of heating is accounted for as the product of cycle time and an assumed $\$ 0.065 / \mathrm{kWh}$ price of electricity. Labor costs are based on the total cycle time, including the time associated with heating the substrate. Table 1 lists specific input cost allowances for the apparatus components and for IC material usage.

TABLE 1. Input Assumptions for the Cost Analysis

\begin{tabular}{|c|c|}
\hline Parameter & Value \\
\hline CdTe module dimensions & $1.2 \mathrm{~m} \times 0.6 \mathrm{~m}$ \\
\hline Number of interconnects & 120 \\
\hline Ag nanoparticle ink use & $0.04 \mathrm{~cm}^{3} /$ module \\
\hline Ag nanoparticle ink price & $\$ 2.78 / \mathrm{g}(\$ 8,340 / \mathrm{L})$ \\
\hline Insulating ink use & $0.02 \mathrm{~cm}^{3} /$ module \\
\hline Insulating ink price & $\$ 0.04 / \mathrm{g}(\$ 41.52 / \mathrm{L})$ \\
\hline Inkjet printing material utilization rate & $99 \%$ \\
\hline Scribe-and-print throughput* & 100 modules/hour \\
\hline Inkjet printer price & $\$ 500,000$ \\
\hline Laser scribe price** & $\$ 754,000$ \\
\hline Heater price & $\$ 120,000$ \\
\hline $\mathrm{T}_{\text {substrate }}$ during scribe $\&$ printing & $150^{\circ} \mathrm{C}$ \\
\hline
\end{tabular}

Specific item cost input data have been aggregated via correspondence and quotations from material suppliers and equipment vendors - multiple of each whenever possible. Company-sensitive information has been anonymized in this report, out of necessity. All of these estimated costs reductions hinge on the assumed development of an actual integrated tool capable of scribing, printing, and ink-curing, with the total capital equipment cost equal to the sum of each process subsystem, plus an additional $20 \%$ added to each component for auxiliary equipment and integration as shown. Additional details of the costs of the various steps involved in the CdTe process flow methodology for cost analysis can be found in previously published work [22-24].

Fig. 9 presents the expected cost reduction in $\$ / \mathrm{m}^{2}$ and $\$ / \mathrm{W}_{\mathrm{p}(\mathrm{DC})}$ possible when employing both 1- and 2-scribe printed ICs in CdTe module manufacturing. The 2015 benchmark costs $\left(2.10 \$ / \mathrm{m}^{2}\right.$ and $\left.0.44 \$ / \mathrm{W}\right)$ assume the traditional P1, P2, and P3 scribe architecture, and $16 \%$ photo-conversion efficiency - accurate representation of characteristic, commercially available, CdTe modules on the market currently [25]. The calculated $\$ / \mathrm{m}^{2}$ cost of production is slightly higher with a 2-scribe IC process $\left(2.51 \$ / \mathrm{m}^{2}\right)$, and lower $\left(1.52 \$ / \mathrm{m}^{2}\right)$ with a printed 1-scribe IC process, compared to the benchmark, $\mathrm{P} 1+\mathrm{P} 2+\mathrm{P} 3$-scribe reference case. Higher $\$ / \mathrm{m}^{2}$ cost with 2-scribe ICs results from added IC material costs (i.e. inks), which add up to an estimated $0.50 \$ / \mathrm{m}^{2}$. However, elimination of two scribing steps and associated toolsets in the conventional fabrication sequence reduces capital and labor costs, and imparts lower a $\$ / \mathrm{m}^{2}$ cost with 1-scribe ICs. Total electricity usage is also reduced in the printed IC processes, even considering the energy required to heat the substrate, since elimination of two process steps decreases total touch-time in 
module production. Lower calculated $\$ / \mathrm{W}$ costs for both 1-scribe and 2-scribe ICs $(0.42$ $\$ / \mathrm{W}$ and $0.43 \$ / \mathrm{W}$, respectively) compared to the benchmark, results primarily from the $2 \%$ relative increase in power achieved with narrower ICs, which reduces the $\$ / \mathrm{W}$ cost of all process steps. Here, it appears that even with a 2-scribe IC process, if $100 \mu \mathrm{m}$ IC width can be achieved, then $\$ 0.01 / \mathrm{W}_{\mathrm{p}(\mathrm{DC})}$ manufacturing cost savings will result.
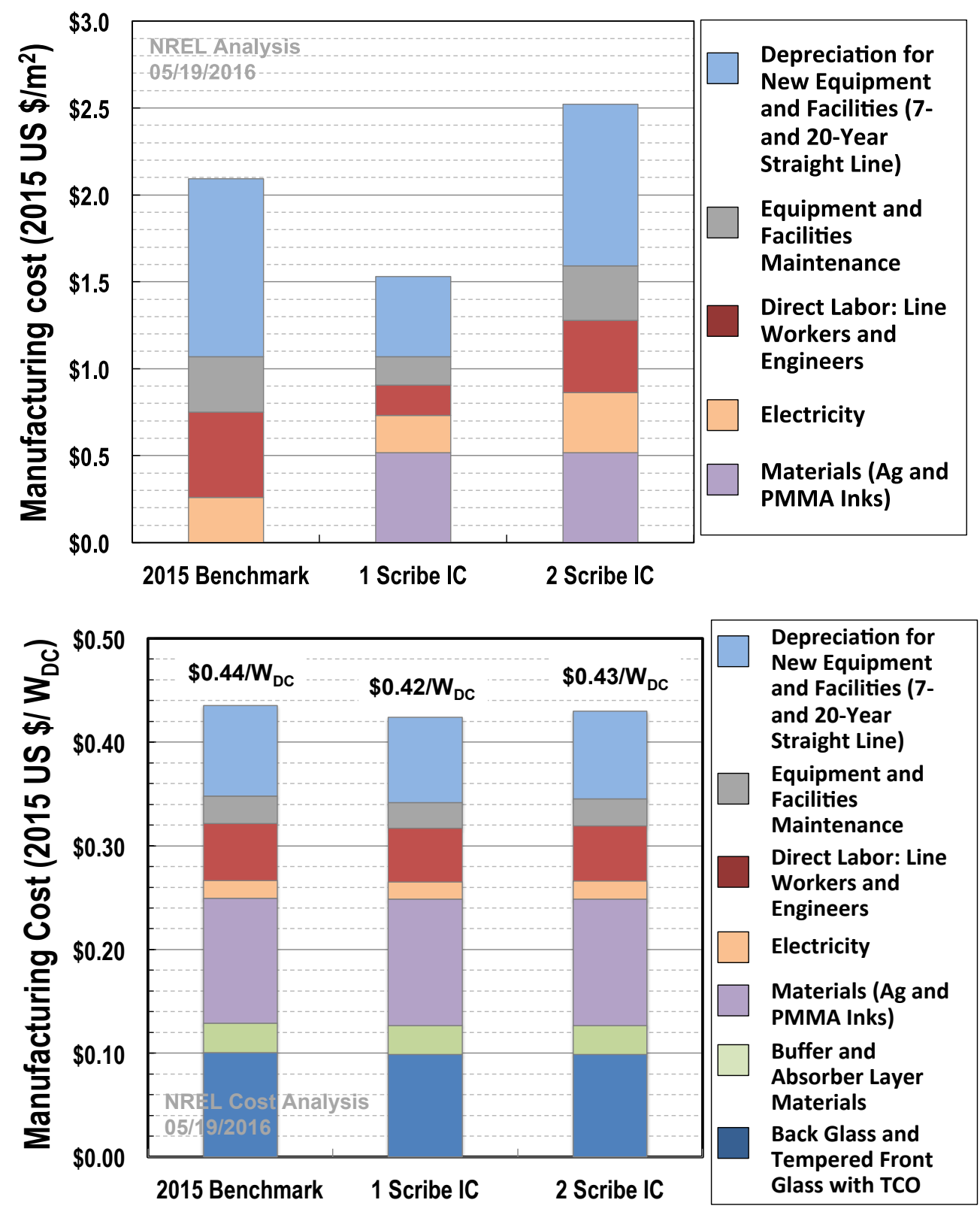

Fig. 9. Estimated manufacturing costs on a $\$ / W$ (top) and $\$ / \mathrm{m}^{2}$ basis (bottom), for CdTe solar cell manufacturing with printed interconnects compared against the 2015 benchmark having the traditional P1, P2, P3 scribe architecture.

Itemized manufacturing cost projections in Fig. 9 assume standard US module manufacturing conditions of $0.6 \mathrm{~m} \times 1.2 \mathrm{~m}$ module size, and $16 \%$ photo-conversion efficiency. Labor cost calculations assume 1.5 employees per MW of production, based on input from industry contacts. Stated costs in Fig. 9(top) omit the cost of the TCO, which is 
assumed equivalent in all cases, whereas Fig. 9(bottom) includes the TCO. Projected costs do not incorporate influence of tax exemptions or tariffs. By this reasoning, use of a 1-scribe or 2-scribe IC convention provides a manufacturing cost savings of $0.02 \$ / \mathrm{W}$ or $0.01 \mathrm{\$} / \mathrm{W}$, respectively, compared to the benchmark case. As these calculations rely on said assumptions, the precise amount of savings achievable using printed ICs is somewhat speculative. However, cost sensitivity analysis shows the general expectation of favorable manufacturing economy to be both robust and justifiable.

Fig. 10 shows the sensitivity of total CdTe module manufacturing costs in $\$ / \mathrm{W}$ to changes in key input parameters associated with a 1-scribe, printed-IC, module manufacturing process by $+/-20 \%$. Again, the reference case for comparison considers conventional module construction, with $\mathrm{P} 1+\mathrm{P} 2+\mathrm{P} 3-$ scribe ICs and an area-specific cost of $2.10 \$ / \mathrm{m}^{2}$, which translates to $0.44 \$ / \mathrm{W}_{\mathrm{DC}}$ with $16 \%$ photo-conversion efficiency. The projected cost using 1-scribe ICs $\left(0.42 \$ / \mathrm{W}_{\mathrm{DC}}\right)$ is found to be most sensitive to production throughput, which the benchmark assumes to be 100 modules per hour based on anonymous industry member surveys. The CdTe module manufacturing cost when using printed ICs remains below that of the conventional P1+P2+P3 scribe IC reference case over a large range of possible input values. Even with $20 \%$ higher cost of $\mathrm{Ag}$ ink, the cost remains below the reference condition. Using this method, it seems a 1 scribe IC process only loses its $\$ / \mathrm{W}_{\mathrm{p}(\mathrm{DC})}$ advantage when the throughput drops from 100 modules per hour to less than 25 modules per hour, the price per integrated scribe-and-print tool (estimated $\$ 1.5$ million) exceeds $\$ 8$ million, or the Ag nanoparticle ink price increases from 2.78 \$/g to $\$ 20 / \mathrm{g}$. Thus, even with a degree of acknowledged uncertainty in the projected prices, a savings of $\$ 0.01$ to $\$ 0.03 / \mathrm{W}_{\mathrm{DC}}$ seems a reasonable expectation.

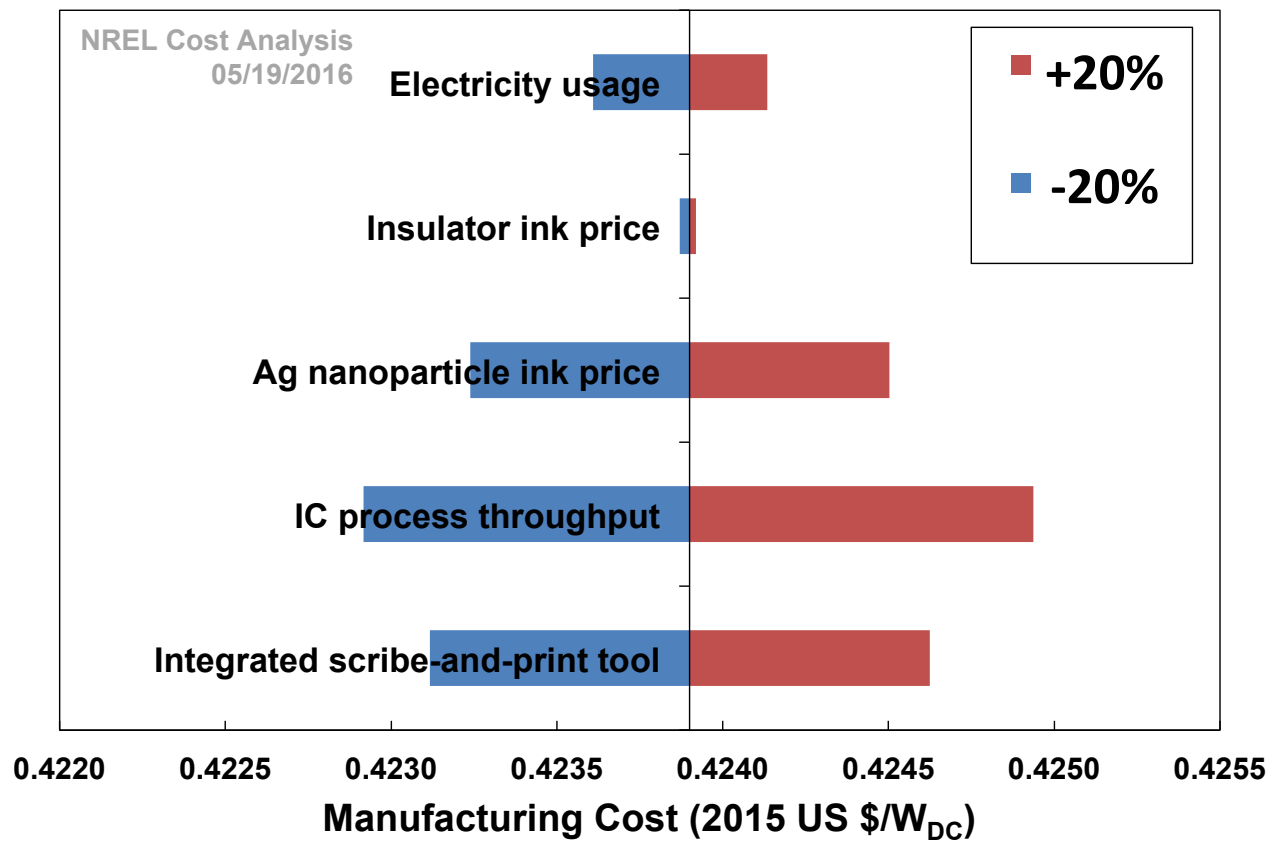

Fig. 10. Sensitivity of projected 1-scribe interconnected CdTe module manufacturing cost to $\pm 20 \%$ changes in input parameter expenses, with assumed module efficiency of $16 \%$.

Cost modeling convincingly shows that printing ICs can lower the manufacturing cost. The 2-scribe architecture, which may limit the minimum achievable IC width to $\sim 100 \mu \mathrm{m}$, can provide an estimated $0.01 \$ / \mathrm{W}$ savings, and $\sim 0.02 \$ / \mathrm{W}$ is achievable if the IC can be made using the suggested 1-scribe geometry. Ultimately, the cost may be reduced even 
further if it becomes possible to eliminate the heater, print both the dielectric and metal at room temperature, and utilize of alternative, non-thermal, ink-curing approaches like UVcuring as reported in Ref. [26]. Lastly, while the recommended use of ultrafast lasers (i.e. ps-pulsed) to avoid shunting, and hence the need for P2-scribing as explained above, may add additional costs (reliable pricing information could only be obtained for ns-pulsed lasers at this time), the input variables sensitivity investigation finds the laser cost may increase up to $10 \mathrm{x}$ before the printed IC process loses a cost advantage. Recognizing a clear cost-reduction case associated with printing IC module fabrication, it is clear why many have launched development activities in this technology.

\section{Summary}

With appropriate dielectric and metal inks, printing module ICs provides a means to increase the geometric fill factor of both substrate and superstrate configuration thin-film $\mathrm{PV}$ modules, and to achieve an estimated manufacturing cost savings of $\$ 0.02 / \mathrm{W}_{\mathrm{p}}$. The cost savings comes from elimination of process steps, which increases throughput and eliminates some capital expense associated with the conventional $\mathrm{P} 1+\mathrm{P} 2+\mathrm{P} 3$ scribe process. Printing the ICs also improves module $\$ / \mathrm{W}_{\mathrm{p}}$ energy economy by increasing power output, since narrower ICs with alternative architecture become possible. Experiments on both CIGS and CdTe solar cells, using aerosol-jetted PMMA and Ag nanoparticle inks to construct printed ICs, demonstrate successful voltage addition. In addition, initial IC narrowing efforts to connect CIGS solar cells with a 2-scribe IC architecture, succeeded at reducing the IC-width to $250 \mu \mathrm{m}$. Ultimately, all of these demonstrations, the provided list of guidelines for selecting dielectric and metal inks, and the numerous advantages of end-of-line IC construction compared to alternate deposition-scribing module-assembly, will continue to drive the uptake of printed ICs in thin-film PV manufacturing.

\section{Acknowledgments}

This work is supported by the Department of Energy through the Bay Area Photovoltaic Consortium under Award Number DE-EE0004946. The authors also acknowledge HelioVolt for providing an abundance of CIGS solar cells, and the NREL CdTe group for providing CdTe cells. Special thanks to Dr. Miguel Contreras, Dr. Matthew Reese, and Dr. Hasitha Mahabaduge for assistance with the CIGS and CdTe cells, and also to Dr. Steve Johnston for assistance with diagnostics and troubleshooting in initial module builds. Additional acknowledgment goes to Linda Lung and the DOE funded Science Undergraduate Laboratory Internship program, which helped to advance the project by addition of talented young researchers, Greg Pach and Talysa Stockert.

\section{References}

[1] J. Hermann, M. Benfarah, S. Bruneau, E. Axente, G. Coustillier, T. Itina, J.F. Guillemoles, P. Alloncle, Comparative investigation of solar cell thin film processing using nanosecond and femtosecond lasers, J. of Appl. Phys. D, 39 (2006) 453-460.

[2] A. Letsch, D. Bartl, M. Diez, R. Gauch, A. Michalowski, R. B. Gmbh, Laser processes for future solar cells, in: Proceedings of SPIE, 8243 (2012) 824317. 
[3] G. Heise, A. Heiss, H. Vogt, H. P. Huber, Ultrafast lasers improve the efficiency of CIS thin film solar cells, Phys. Procedia, 39, (2012) 702-708.

[4] G. Heise, A. Borner, M. Dickmann, M. Englmaier, A. Heiss, M. Kemnitzer, J. Konrad, R. Moser, J. Palm, H. Vogt, H. P. Huber, "Demonstration of the monolithic interconnection on CIS solar cells by picosecond laser structuring on 30 by $30 \mathrm{~cm} 2$ modules" Prog. Photovolt.: Res. Appl., 15 (2014) 2552.

[5] E. Markauskas, P. Gecys, A. Zemaitis, M. Gedvilas, G. Raciukaitis, Validation of monolithic interconnection conductivity in laser scribed CIGS thin-film solar cells, Sol. Energy, 120, (2015) 35-43.

[6] B. Reinhold, M. Scmid, D. Greiner, M. Schule, D. Kieven, A. Ennauoi, M. C. Lux-Steiner, Monolithically interconnected lamellar $\mathrm{Cu}(\mathrm{In}, \mathrm{Ga}) \mathrm{Se} 2$ micro solar cells under full white light concentration, Prog. Photovolt.: Res. Appl., (2015) 2611.

[7] S. Kirner, S. Neubert, C. Schultz, O. Gabriel, B. Stannowski, B. Rech, R. Schlatmann, Quadruple-junction solar cells and modules based on amorphous and microcrystalline silicon with high stable efficiencies" Jpn. J. Appl. Phys., 54 (2015) 08KB03.

[8] K. Thalheimer, Procedure for producing an integrated system of thin-film solar cells connected in series, US Patent No. 4758526 (1988).

[9] A. Brunton, Method and apparatus for dividing thin film device into separate cells, US Patent No. 9130094 B2 (2011).

[10] M. L. Crozier, P. Adamson, A. Brunton, S. Henley, J. D. Shephard, G. Kartopu, S. Irvine, P. M. Kaminski, J. M. Walls, Recent developments toward a one step thin-film PV interconnection process using laser scribing and inkjet printing, in: Proceedings of the 41st IEEE PVSC (2014) 2784-2788.

[11] M.F.A.M. van Hest, H.S. Platt, Photovoltaic sub-cell interconnects, US Patent Application US 2014/0345673 A1.

[12] S. Jasen, C. P. Kotarba, J. Pappas, R. Powell, Y. Roussillon, E. Van Nortwick, and C. Wickersham, "Photovoltaic device interconnection and method of manufacturing" US Patent Application 2015/0263197 A1 (2015).

[13] J. D. Fields, M. S. Dabney, V. P. Bollinger, M. F. A. M. van Hest, “Printed monolithic interconnects for photovoltaic applications" in: Proceedings of the 40th IEEE PVSC (2014) 162-165.

[14] A. Zimmermann, Monoscribe: Roll-to-Roll Monolithic Interconnection of Customizable Thin-Film Solar Modules, presented at Solar-ERA Information and Match-Making event, in Dusseldorff, Austria, Feb 24 (2016).

[15] S. Khan, L. Lorenzelli, R. S. Dahiya, Technologies for printing sensors and electronics over large flexible substrates: A review, J. IEEE Sens., 15, 3164 (2015).

[16] M. Singh, H. M. Haverinen, P. Dhagat, G. E. Jabbour, Inkjet printing-process and its applications, Adv. Mater., 22 (2010) 673-685.

[17] J. H. Yoon, J. K. Park, W. M. Kim, J. W. Lee, H. Pak, and J. H. Jeong, “Characterization of efficiency-limiting resistance losses in monolithically integrated $\mathrm{Cu}(\mathrm{In}, \mathrm{Ga}) \mathrm{Se}_{2}$ solar modules" Sci. Rep., 5 (2015) 7690.

[18] H. P. Mahabaduge, W. L. Rance, J. M. Burst, M. O. Reese, D. M. Meysing, C. A. Wolden, J. Li, J. D. Beach, T. A. Gessert, W. K. Metzger, S. Garner, T. M. Barnes, High-efficiency, flexible CdTe solar cells on ultra-thin flexible glass, Appl. Phys. Lett., 106 (2015) 133501.

[19] P. A. Hersh, C. J. Curtis, M. F. A. M. van Hest, J. J. Kreuder, R. Pasquarelli, A. Miedaner, and D. S. Ginley, Inkjet printed metallizations for $\mathrm{Cu}\left(\mathrm{In}_{1-\mathrm{x}} \mathrm{Ga}_{\mathrm{x}}\right) \mathrm{Se}_{2}$ photovoltaic cells Prog. Photovolt.: Res. Appl., 19 (2011) 973. 
[20] N. G. Dhere, A. Kaul, J. Shimada, A. Kar, Review of laser material interaction to improve CIGSeS thin film solar cells, in: Proceedings of SPIE 8473 (2012) 84730G.

[21] M. W. Rowell, M. D. McGehee, Transparent electrode requirements for thin film solar cell modules, Energy \& Environ. Sci., 4 (2011) 131-134.

[22] R. Fu, T. L. James, M. Woodhouse, Economic measurements of polysilicon for the photovoltaics industry: Market competition and manufacturing competitiveness, IEEE J. Photovolt., 5 (2015) 515-524.

[23] M. Woodhouse, A. Goodrich, R. Margolis, T. James, R. Dhere, T. Gessert, T. Barnes, R. Eggert, D. Albin, Perspectives on the pathways for cadmium telluride photovoltaic module manufacturers to address expected increases in the price for tellurium, Sol. Energy Mater. Sol. Cells, 115, (2013) 199-212.

[24] A. Goodrich, P. Hacke, Q. Wang, B. Sopori, R. Margolis, T. L. James, and M. Woodhouse, A wafer-based monocrystalline silicon photovoltaics road map: utillizing known technology improvement opportunities for further reductions in manufacturing costs, Sol. Energy Mater. Sol. Cells, 114 (2013) 110-135.

[25] S. Krum, "First Solar achieves yet another cell conversion efficiency world record," in: First Solar Media Release (2016).

[26] Z. Li, D. S. Tan, Q. L. Ren, Q. Fu, Y. F. Xu, Y. F. Tao, Synthesis and properties of UVcurable polysiloxane methacrylate obtained by one-step method, Chn. J. Polymer Sci., 31 (2013) 363-370. 